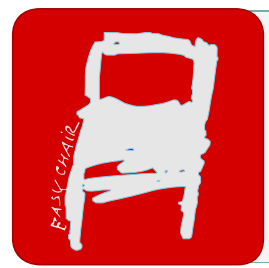

EPiC Series in Health Sciences

\title{
Can Knee Stability at 0 and 90 Degrees Accurately Predict Mid-Flexion Stability in Total Knee Arthroplasty?
}

\author{
Bertrand Kaper ${ }^{1,2}$ \\ 1 Orthopaedic Specialists of Scottsdale \\ 2 Scottsdale, AZ USA \\ bkaper@drkaper.com
}

\begin{abstract}
The goal of this study was to utilize the NAVIO robotic-assisted (RA)-TKA technique to assess whether a knee that is well-balanced at 0 and $90^{\circ}$ is also well balanced in mid-flexion. Using a $3 \mathrm{~mm}$ threshold to define soft-tissue balance, results demonstrated that $11.5 \%$ of knees studied could be expected to be unstable in the mid-flexion arc $\left(15-75^{\circ}\right)$ despite being well-balanced at the static poses at 0 and $90^{\circ}$.
\end{abstract}

\section{Introduction}

Mid-flexion stability has been recognized a potential failure etiology in total knee arthroplasty (TKA) $)^{1,2,3,4}$. The assessment of soft tissue balance in conventionalinstrumented $(\mathrm{Cl})$ TKA is typically limited to an objective assessment at the static poses at zero and ninety degrees. Assessment of mid-flexion balance is, by default, driven primarily by the subjective feel of the surgeon. Surgeon-defined assessment of soft tissue stability, however, has been shown to be a poor predicter of true soft tissue balance ${ }^{5}$. Furthermore, due to the constraints of conventional-instrumented total knee techniques, the ability to make fine-tuning adjustments to bone resection to reduce the risk of mid-flexion instability are limited ${ }^{6,7}$. An assumption is therefore necessary that a knee that is well-balanced at 0 and $90^{\circ}$ is also well-balanced in mid-flexion. With robotic assisted (RA) TKA techniques, an objective assessment of soft tissue balance throughout the arc of motion is feasible. 
The goal of this study was to utilize the NAVIO RA-TKA technique to assess whether a knee that is well-balanced at 0 and $90^{\circ}$ is also well balanced in mid-flexion.

\section{Methods}

Starting in August 2019, a longitudinal data collection series was initiated in a singlesurgeon total joint arthroplasty registry. Eighty-seven patients undergoing total knee arthroplasty with the NAVIO 7.0 RA-TKA technique were enrolled. Baseline demographic information for all cases was recorded, including gender, age, and BMI. Post-operative data was recorded, including limb alignment and component position.

Utilizing the planning tools inherent to the NAVIO 7.0 technique, surface mapping the distal femur and proximal tibia was performed to generate an "imageless", static 3-dimensional model of the knee. A preliminary, measured resection TKA model, as dictated by the manufacturer's stated surgical technique, was then generated based on bone resection depths. All knees were then taken through a 0-90 "stressed" range of motion- during which both the medial and lateral compartments were subjected to tensile, distractive forces. This manual technique utilized a posterior cruciate ligament (PCL) Hohmann retractor to achieve joint distraction. Based on simulated joint distraction with a calibrated tensioner, the technique allows consistently applied force of approximately $120 \mathrm{Nm}$ to measure soft tissue laxity. The medial and lateral displacements, as a measure of coronal soft tissue laxity or tautness/tightness, were thereby recorded. These measurements were overlaid on the measured resection model.

In the planning stage, adjustments were then made to the surgical plan to balance the medial and lateral soft tissues to $<3 \mathrm{~mm}$ at the static poses of 0 and $90^{\circ}$. This was accomplished via adjustments in femoral and tibial prosthetic component size, position and orientation in the coronal, sagittal and transverse planes [Image 1].

Joint laxity in mid-flexion (between $15-75^{\circ}$ ) was then recorded, differentiating those cases that were well-balanced $(<3 \mathrm{~mm})$ or not well-balanced $(>3 \mathrm{~mm})$ [Image 2]. This completed the data acquisition to address the question posed in this study. 


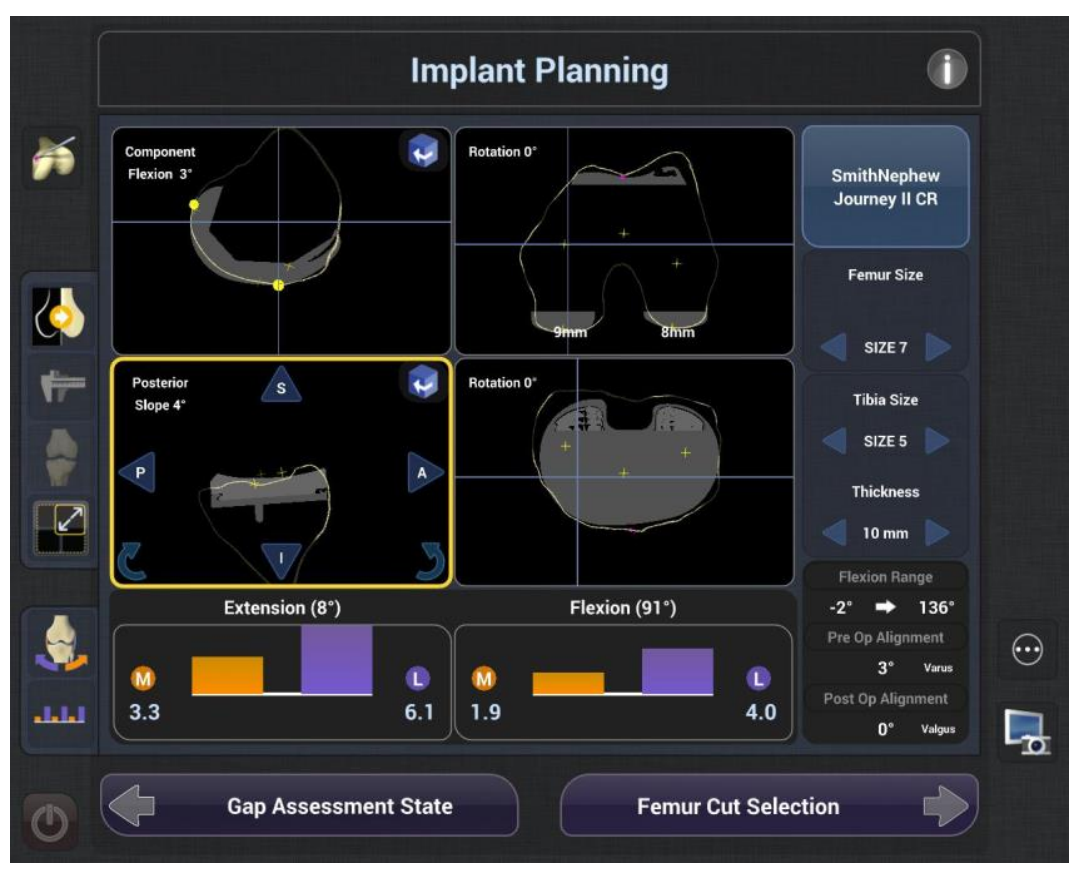

Image 1. Planning of implant components in 3D space for joint balance from 0 to $90^{\circ}$.

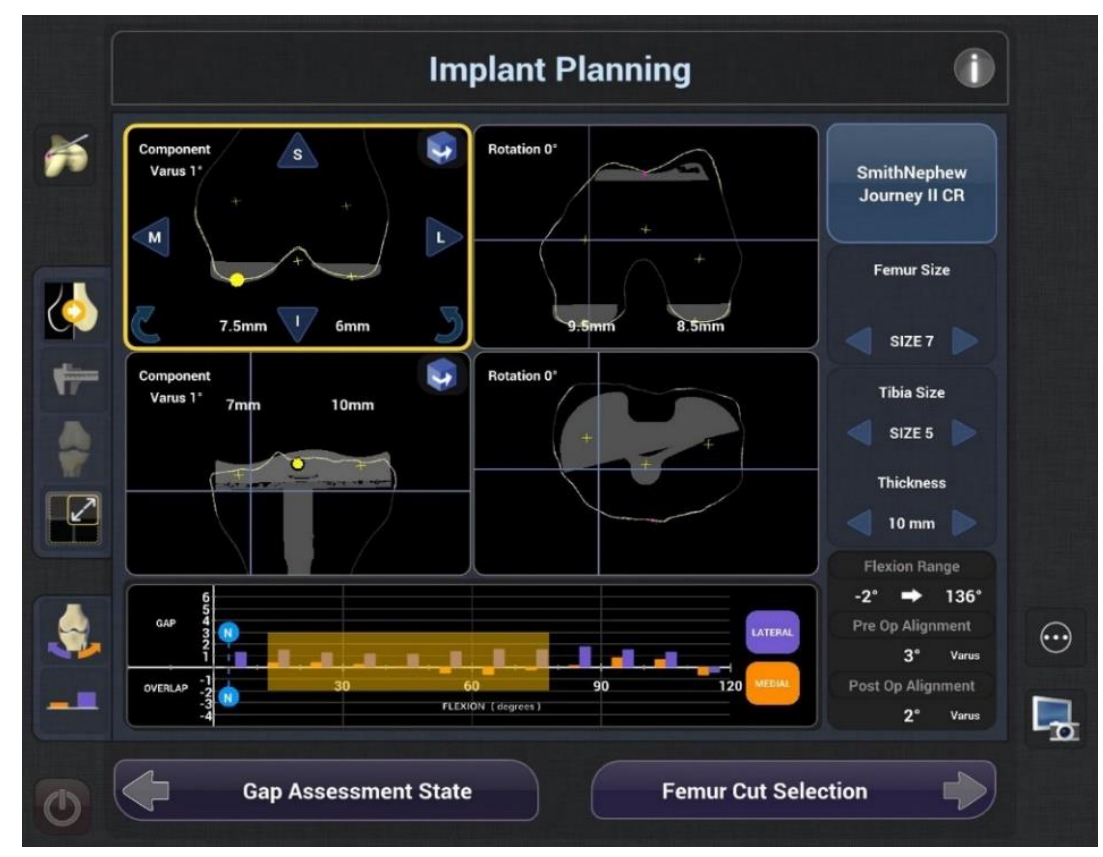

Image 2. NAVIO 7.0 Implant Planning incorporating an assessment, in mid-flexion, of medial and lateral soft tissue balance. 
For those cases in which mid-flexion instability was noted intra-operatively, changes to the surgical plan were subsequently made. Typically, reassessment of the joint line height and/or adjustment to the anterior/posterior of the position of the femoral component allowed correction of any mid-flexion instability [Image 3].

NAVIO 7.0 software and the Journey II total knee prosthesis was used for all cases.

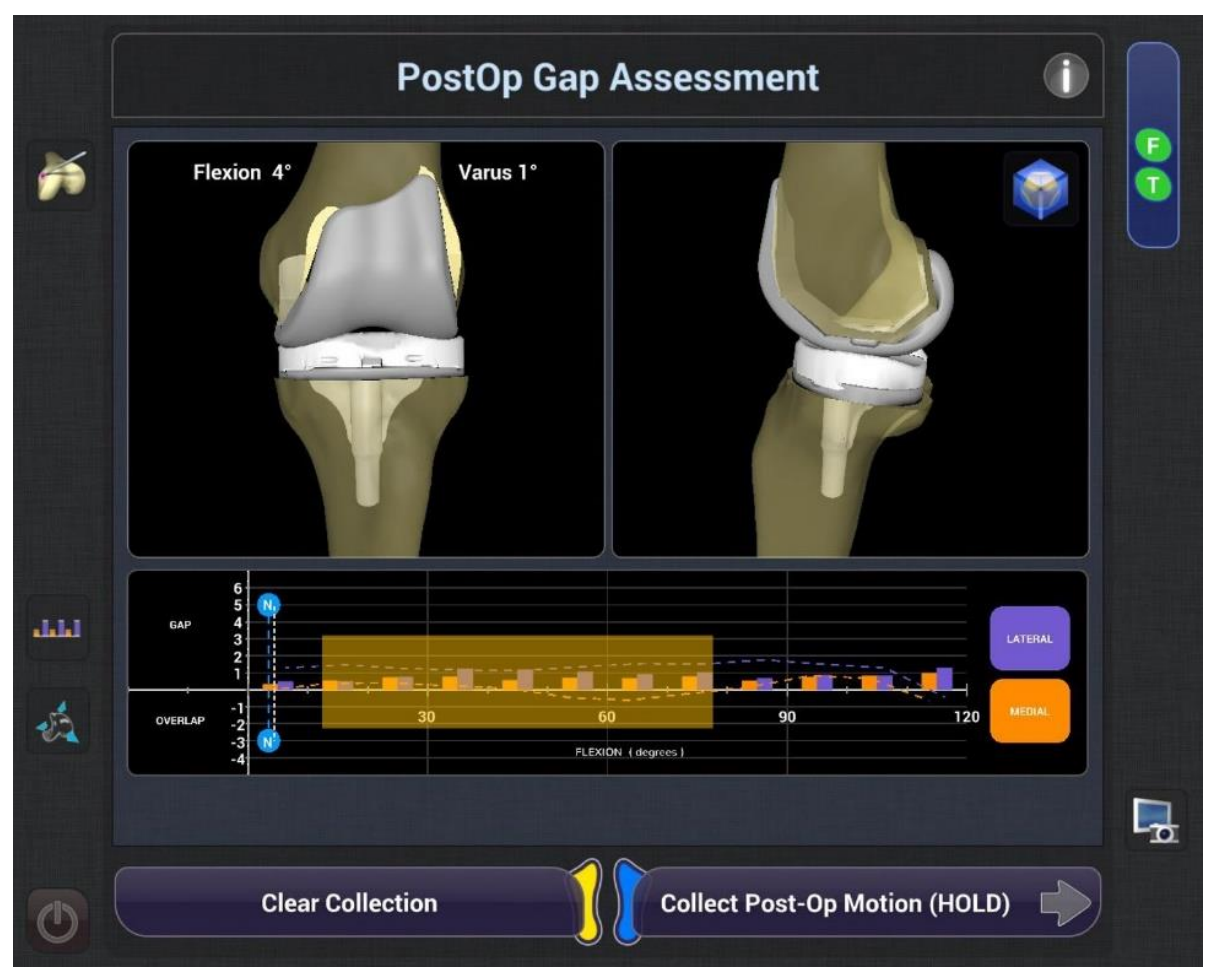

Image 3. Planned gaps from image 2, are represented as dashed lines, and final achieved gaps are represented by bars, after the placement of components post bone cutting. 


\section{Results}

Baseline demographic data demonstrated average patient age of 69.7 years (range 51-85 years); average patient BMI of 29.6 (range 19-48); 34 male (39\%) and 53\% female patients (61\%).

Post-operative radiographic evaluation demonstrated average mechanical axis at the knee of $179.8^{\circ}$. Radiographic measurements included an average distal femoral angle of $93.6^{\circ}$ (range $92-96^{\circ}$ ) and femoral flexion angle of $2.2^{\circ}$ (range $0-5^{\circ}$ ); with a proximal tibial angle of $89.3^{\circ}$ (range $87-91^{\circ}$ ) and posterior tibial slope of $3.7^{\circ}$ (range $3-6^{\circ}$ ).

Intra-operative soft tissue balancing ( $<3 \mathrm{~mm}$ laxity) at the 0 and $90^{\circ}$ poses was accomplished via adjustments in femoral and tibial prosthetic component size, position and orientation in the coronal, sagittal and transverse planes in all cases. Once balance was achieved at 0 and $90^{\circ}$, an assessment of stability in mid-flexion was recorded.

Ten of the 87 cases (11.5\%) failed to demonstrate soft tissue balance in mid-flexion (between $15^{\circ}$ and $75^{\circ}$ ), with either medial or lateral laxity greater than the $3 \mathrm{~mm}$ threshold.

\section{Discussion/Conclusion}

Patient reported outcome measures (PROM's) have consistently demonstrated dissatisfaction rates between $10-20 \%$ following TKA surgery ${ }^{8,9}$. Identifying the cause of this relatively high dissatisfaction rate has proven elusive despite multiple advances in implant design, material science and surgical techniques. Specific to the innovations in surgical technique, technology that has focused on the static/bony aspect of TKA surgery-i.e. computer navigation and patient-specific instrumentation (PSI), has failed to consistently improve TKA PROM's. Technology that has addressed the soft tissue balance, i.e. kinetic sensors, however, have shown promise in improving PROM's.

The focus of TKA primarily as a soft tissue operation has led to concern that instability, even to subtle degrees, may be contributing to patient dissatisfaction. Mid-flexion instability, or alternatively described as lack of mid-flexion balance, has been identified as such a failure etiology. The focus of this study was to gather objective information about mid-flexion soft tissue balance available with the use of RA-TKA technology.

Joint laxity $>3 \mathrm{~mm}$ was chosen as the threshold to define whether the soft tissues were balanced or not. This decision was based on previous publications assessing soft tissue laxity in both healthy knees and knees following TKA ${ }^{10-12}$. 
Using this $3 \mathrm{~mm}$ threshold to define soft-tissue balance, results demonstrated that $11.5 \%$ of knees studied could be expected to be unstable in the mid-flexion arc $\left(15-75^{\circ}\right)$ despite being well-balanced in the static poses at 0 and $90^{\circ}$. This data challenges the assumption that a knee that is well-balanced in the static poses of $0^{\circ}$ and $90^{\circ}$ is also consistently wellbalanced in mid-flexion.

With the addition of robotic-assisted NAVIO technology, an intra-operative assessment of mid-flexion balance can be made. If mid-flexion imbalance is noted, adjustments can be made to the surgical plan (i.e. ensuring joint line accuracy and/or minimizing anterior positioning of the femoral component) to reduce mid-flexion laxity. Potential mid-flexion instability can hereby be identified proactively and changes to the surgical plan be made prior to definitive bone resection.

Further study will be necessary to determine whether the PROM's associated with patients for whom a well-balanced mid-flexion arc was achieved reflect improved outcomes.

\section{References}

${ }^{1}$ Midflexion instability in primary total knee replacement: a review. Manjunath Ramappa' SICOT J. 2015; 1: 24.

${ }^{2}$ Intraoperative assessment of midflexion laxity in total knee prosthesis. Minoda Y, Nakagawa S, Sugama R, Ikawa T, Noguchi T, Hirakawa M, Nakamura H. Knee. 2014 Aug;21(4):810-4.

${ }^{3}$ Total knee arthroplasty using anatomic alignment can produce mid-flexion laxity. Incavo SJ, Schmid S, Sreenivas K, Ismaily S, Noble PC. Clin Biomech. 2013 Apr;28(4):429-35.

${ }^{4}$ Midflexion Laxity After Implantation Was Influenced by the Joint Gap Balance Before Implantation in TKA. Minoda Y, Nakagawa S, Sugama R, Ikawa T, Noguchi T, Hirakawa M. J Arthroplasty. 2015 May;30(5):762-5.

${ }^{5}$ How Accurately Can Soft Tissue Balance Be Determined in Total Knee Arthroplasty? MacDessi SJ et al. J Arthroplasty 2019 Feb; 34(2):P290-294.

${ }^{6}$ Raising the Joint Line in TKA is Associated With Mid-flexion Laxity: A Study in Cadaver Knees. Luyckx T, Vandenneucker H, Ing LS, Vereecke E, Ing AV, Victor J. Clin Orthop Relat Res. 2018 Mar;476(3):601-611. 
${ }^{7}$ A Computer Model of Mid-Flexion Instability in a Balanced Total Knee Arthroplasty. Evangelista PF, Scott K, Laster BA, Lenz NM, Sheth P, Schwarzkopf R. J Arthroplasty. 2018 July;33(7): S265-9.

${ }^{8}$ Patient satisfaction after total knee arthroplasty: who is satisfied and who is not? Bourne RB, Chesworth BM, Davis AM, Mahomed NN, Charron KD. Clin Orthop Relat Res. 2010 Jan;468(1):57-63.

${ }^{9}$ Predicting dissatisfaction following total knee replacement: a prospective study of 1217 patients.

Scott $C^{1}$, Howie CR, MacDonald D, Biant LC. J Bone Joint Surg Br. 2010 Sep;92(9):1253-8.

${ }^{10}$ Accuracy of Soft Tissue Balancing in Total Knee Arthroplasty. Griffin FM, Insall JM, Scuderi G. J Arthroplasty 2000 Dec; 15(8):970-74.

${ }^{11}$ Soft Tissue Tension in Total Knee Arthroplasty. Asano et al. J Arthroplasty 2004 Aug;19(5):558-61

${ }^{12}$ Navigation as a Predictor of Soft Tissue Release During 90 Cases of Computer-assisted Total Knee Arthroplasty. Saragaglia D et al. Orthop 2006 Oct;29(10):S137-38. 